\title{
Conventional mechanical ventilation in pediatrics
}

\author{
Alexandre T. Rotta, ${ }^{1}$ David M. Steinhorn²
}

\begin{abstract}
Objective: To review the various challenges of providing mechanical ventilation to pediatric patients with diseases of increased airway resistance, diseases of abnormal lung compliance or normal lungs.

Sources: Original data from our pediatric intensive care unit and animal research laboratory. Relevant articles included in the MEDLINE electronic database during the last 10 years. Also included were book chapters and definitive studies, as judged by the authors, in the fields of asthma, acute respiratory distress syndrome, mechanical ventilation, ventilator-induced lung injury and permissive hypercapnia.
\end{abstract}

Summary of the findings: Mechanical ventilation of patients with diseases of increased airway resistance should center on avoidance of dynamic hyperinflation, allowing complete exhalation prior to the initiation of a subsequent breath and permissive hypercapnia. Positive end-expiratory pressure should be used sparingly to prevent atelectasis and facilitate synchrony in spontaneously breathing patients. Mechanical ventilation of patients with diseases of abnormal lung compliance should take into consideration the inhomogeneous distribution of lung disease. Focus should be on avoidance of volutrauma and atelectrauma that could result in ventilator-associated lung injury.

Conclusions: The last decade was marked by significant advances in the management of pediatric respiratory failure. The choice of mechanical ventilation strategy can significantly influence the subsequent course of lung injury. Mechanical ventilation can no longer be viewed simply as a harmless support modality that is employed to keep patients alive while disease-specific treatments are used to ameliorate the underlying pathology.

J Pediatr (Rio J). 2007;83(2 Suppl):S100-108: Mechanical ventilation, acute lung injury, acute respiratory distress syndrome, status asthmaticus, permissive hypercapnia.

\section{Introduction}

The use of mechanical ventilation is widespread in Pediatric Intensive Care Units throughout the world as a central strategy in the management of patients with acute and chronic respiratory failure. Although the support of patients with normal, healthy lungs, such as those of patients with neuromuscular respiratory failure or following general anesthesia for elective procedures, can generally be accomplished without difficulty, the ventilation of pediatric patients with serious pulmonary diseases poses a much more difficult challenge to the clinician. With greater severity of lung disease, the risk of secondary injury from the ICU therapies themselves increases.

Mechanical ventilation can no longer be viewed simply as a harmless support modality that is employed to keep patients alive while disease-specific treatments are used to ameliorate the underlying pathology. ${ }^{1}$ We now know that the choice of mechanical ventilation strategy can significantly influence the subsequent course of lung injury. Ventilation strategies that produce regional hyperinflation and the cyclic

1. MD, FAAP. Associate professor, University of Texas Medical Branch at Galveston, Galveston, TX, USA. Pediatric intensivist, Department of Anesthesiology and Critical Care Medicine, Driscoll Children's Hospital, Corpus Christi, TX, USA.

2. MD. Professor of Pediatrics, Northwestern University Feinberg School of Medicine, Evanston, IL, USA. Pulmonary and Critical Care Medicine, Children's Memorial Hospital, Chicago, IL, USA.

Suggested citation: Rotta AT, Steinhorn DM. Conventional mechanical ventilation in pediatrics. J Pediatr (Rio J). 2007;83(2 Suppl):S100-108.

doi 10.2223/JPED. 1617 
opening and closing of bronchoalveolar units have been linked to ventilator-associated lung injury. ${ }^{2-4}$ In contrast, a strategy that incorporates the basic principles of lung protective ventilation can hasten recovery and minimize pulmonary morbidity with its attendant mortality. ${ }^{5}$

\section{Mechanical ventilation of healthy lungs}

Mechanical ventilation of patients with healthy lungs, normal pulmonary compliance and airway resistance can generally be accomplished without difficulty. Regardless of the mode of ventilation used (pressure or volume limited) the tidal volume is distributed fairly evenly throughout the healthy lung during inspiration due to the homogeneous compliance among various lung regions. Passive exhalation also occurs without impediment and the flow of gas mimics that observed during effortless spontaneous breathing. So tolerant is the healthy lung to mechanical ventilation that supraphysiologic tidal volumes $(10-15+\mathrm{mL} / \mathrm{kg})$ and moderately high pressures are well tolerated for periods of minutes to hours with little injury to the lung. This approach has been utilized during elective anesthesia for many years.

Specific ventilator settings vary from patient to patient according to age, size and body habitus. Nevertheless, an effective tidal volume of 8 to $10 \mathrm{~mL} / \mathrm{kg}$ of ideal body weight is generally appropriate for most patients. When a pressure-limited mode is chosen, the peak inspiratory pressure should be set to generate tidal volumes within that same range, which can usually be accomplished with peak inspiratory pressures of approximately $20 \mathrm{cmH}_{2} \mathrm{O}$, or even lower in young patients. Because these ventilation strategies tend to operate in a more linear portion of the pressure-volume curve, changes in pressure tend to be associated with directly proportional changes in the delivered tidal volume. Positive end-expiratory pressure (PEEP) should be applied to decrease the likelihood of dependent atelectasis in intubated patients, particularly in those patients unable to sigh or spontaneously breathe along with assisted breaths. The inspiratory times are generally close to those of age-appropriate spontaneous breathing, such as approximately 0.4 seconds for newborns, 0.6 seconds for toddlers, 0.8 seconds for older children and 1 second for teenagers and young adults. The mandatory respiratory rate is inversely related to age and size and should be set to generate a minute volume that results in normocapnia, as measured by serial blood gas analysis, transcutaneous monitoring or through continuous end-tidal capnography.

\section{Mechanical ventilation in diseases of abnormal airway resistance}

Diseases of abnormal airway resistance are characterized by an impediment to gas flow that tends to be more significant during the expiratory phase of the respiratory cycle. As such, these patients present with prolonged expiratory times, expiratory wheezing and dynamic pulmonary hyperinflation. ${ }^{6}$ The increased airway resistance may result from bronchospasm, mucosal edema, mucous plugging, intraluminal debris, extrinsic compression, or, as it is often the case, a combination of these factors.

Asthma is the prototypical disease of increased airway resistance in the pediatric population. ${ }^{6}$ Status asthmaticus and its more severe counterpart near-fatal asthma are common causes of admission to the Pediatric Intensive Care Unit (PICU), although only a minority of these patients requires mechanical ventilation support. ${ }^{6}$ The incidence of asthma-related respiratory failure requiring mechanical ventilation is difficult to determine due to variability in diagnostic criteria and reporting practices. Nonetheless, as many as $36 \%$ of adult patients admitted to an inner-city medical ICU with severe asthma require invasive mechanical ventilation. ${ }^{7}$ This figure appears to be significantly lower for children, considering that only 22 out of 237 (10.2\%) patients treated for near-fatal asthma underwent mechanical ventilation in the PICU at the Women and Children's Hospital of Buffalo over a two-year period, and that 14 out of 163 $(8.6 \%)$ patients required intubation during a recent study elsewhere. ${ }^{8}$

The goal of mechanical ventilation in acute asthma is to reverse hypoxemia (if present), relieve respiratory muscle fatigue and maintain a level of alveolar ventilation compatible with an acceptable $\mathrm{pH}$, while avoiding iatrogenic hyperinflation and levels of intrathoracic pressure that could negatively impact cardiac output. ${ }^{9}$ Therefore, the choice of mechanical ventilator settings must take into consideration the significant derangements of lung mechanics that are seen with severe acute asthma. Inexpert attempts to achieve a normal $\mathrm{PaCO}_{2}$ would require fast respiratory rates, high minute volumes and very high airway pressures, which are associated with the development of barotrauma (pneumothorax and pneumomediastinum), and increased mortality. ${ }^{10-12}$

A paradigm shift in the ventilatory management of asthmatic patients occurred with the introduction of Darioli \& Perret's strategy of controlled hypoventilation. ${ }^{1}$ This strategy resulted in no mortality in 34 episodes of mechanical ventilation in 26 patients, and significantly lower complication rates in comparison to historical controls. ${ }^{13}$ This approach consists of tidal volumes between 8 and $12 \mathrm{~mL} / \mathrm{kg}$ and peak airway pressures lower than $50 \mathrm{cmH}_{2} \mathrm{O}$. Tidal volumes should be further reduced if the desired peak pressure limit cannot be respected, and higher $\mathrm{PaCO}_{2}$ measurements should be tolerated. A similar approach using 
respiratory rates lower than 12 breaths per minute, tidal volumes between 8 and $12 \mathrm{~mL} / \mathrm{kg}$, peak inspiratory pressures of 40 to $45 \mathrm{cmH}_{2} \mathrm{O}$, and permissive hypercapnia has also been associated with very few complications and no mortality or long-term morbidity in 19 mechanically ventilated children with near-fatal asthma. ${ }^{14}$

The modes of ventilatory support for patients with severe acute asthma can be divided into pressure and volume preset. No definitive evidence exists to suggest that one particular mode of ventilation is superior to the other. However, to safely ventilate the asthmatic patient, one must understand the characteristics of each mode. Pressure control modes employ a decelerating gas flow and have the advantage of ensuring that a particular inspiratory pressure limit is respected. The main disadvantage of pressure control modes is that tidal volumes can vary greatly with changes in airway resistance and the state of pulmonary hyperinflation. In patients with bronchospasm, airway resistance variations throughout the lung lead to variable time constants with inconsistent time for air entry and exit. In contrast, volume control modes deliver a constant tidal volume, provided there is no significant air leak around the endotracheal tube. An added advantage of volume control is that it allows for comparison of peak inspiratory pressure and plateau pressure measurements (peak-to-plateau pressure difference), which can serve as an indirect longitudinal indicator of airway resistance and response to therapy. ${ }^{6}$ For these measurements, the plateau pressure (static end-inspiratory pressure) is obtained by performing an inspiratory hold (a feature available on most modern ventilators) and is then compared to the peak inspiratory pressure (Figure 1). An increasing peak-to-plateau pressure difference indicates increasing airway resistance, while a decreasing peak-toplateau pressure difference suggests response to therapy. A disadvantage of volume control ventilation is that patients can develop very high lung volumes if exhalation is incomplete, since tidal volumes remain constant breath to breath. This condition is called breath stacking and results in so-called auto-PEEP when breaths are unable to completely empty. A relatively new mode of ventilation termed pressure-regulated volume control (PRVC), available on some ventilators, combines some of the advantages of pressure control and volume control, including optimal inspiratory gas flow, assured tidal volumes and limited airway pressures.

The use of PEEP in intubated asthmatics has been the focus of controversy. Externally applied PEEP may, in theory, benefit patients with expiratory flow limitation caused by dynamic compression of small airways by shifting the equal pressure point distally, stenting collapsed or severely

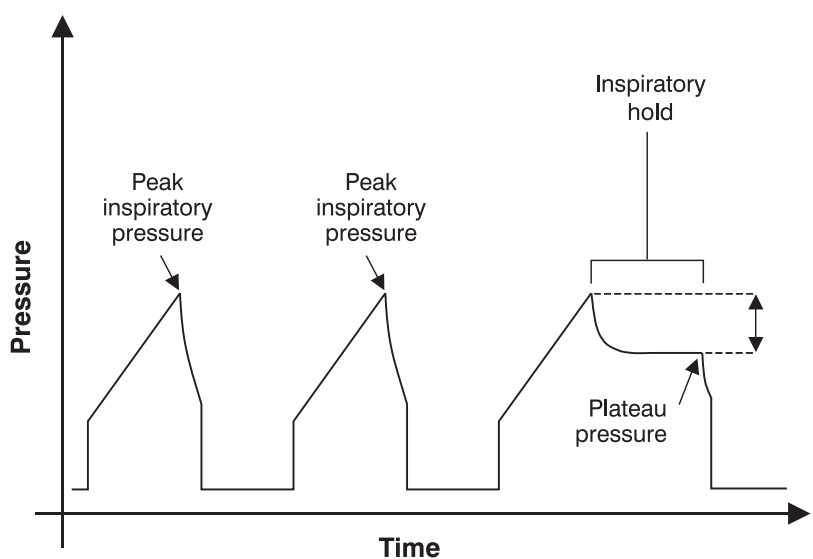

Figure 1 - Schematic representation of the airway pressure waveform over time during volume control ventilation. The peak-to-plateau pressure difference (double-headed arrow) is obtained after an inspiratory hold by comparing the peak pressure and the measured plateau pressure

narrowed airways and enabling decompression of upstream alveoli. ${ }^{15}$ The application of low levels of PEEP which are, by definition, lower than the level of auto-PEEP, may also relieve dyspnea by facilitating ventilator triggering and synchronization for intubated patients capable of taking spontaneous breaths. ${ }^{15,16}$ However, as elegantly demonstrated by Tuxen, ${ }^{17}$ the use of PEEP in chemically paralyzed patients with severe airflow obstruction has been uniformly associated with higher lung volumes, increased airway and intrathoracic pressures and circulatory compromise. In practice, the benefits of applying PEEP, e.g. minimizing atelectasis, stenting airways, must be balanced with the tendency to produce higher lung volumes and the potential for barotrauma.

Our personal preference is to use the synchronized intermittent mandatory ventilation (SIMV) mode, employing tidal volumes of 8 to $12 \mathrm{~mL} / \mathrm{kg}$, to generate peak inspiratory pressures $<35 \mathrm{cmH}_{2} \mathrm{O}$ and plateau pressures $<30 \mathrm{cmH}_{2} \mathrm{O}$. Tidal volumes may need to be further reduced to achieve these parameters in individual patients. Respiratory rate is initially set between 6 and 12 breaths per minute and inspiratory time is set between 1 and 1.5 seconds, allowing for expiratory times between 4 and 9 seconds. The respiratory rate is adjusted according auscultation and respiratory flow-time curves so as to ensure that complete exhalation has occurred before the next breath is delivered (Figure 2). PEEP is set at zero for the patient under neuromuscular blockade. With clinical improvement, neuromuscular blockade is discontinued and trigger sensitivity of the ventilator is optimized for spontaneous breaths. A low level of PEEP (lower than the measured auto-PEEP and never in excess of $8 \mathrm{cmH}_{2} \mathrm{O}$ ) is applied to 
facilitate synchronization between patient and machine, and spontaneous breaths are aided by the application of pressure support. ${ }^{6}$
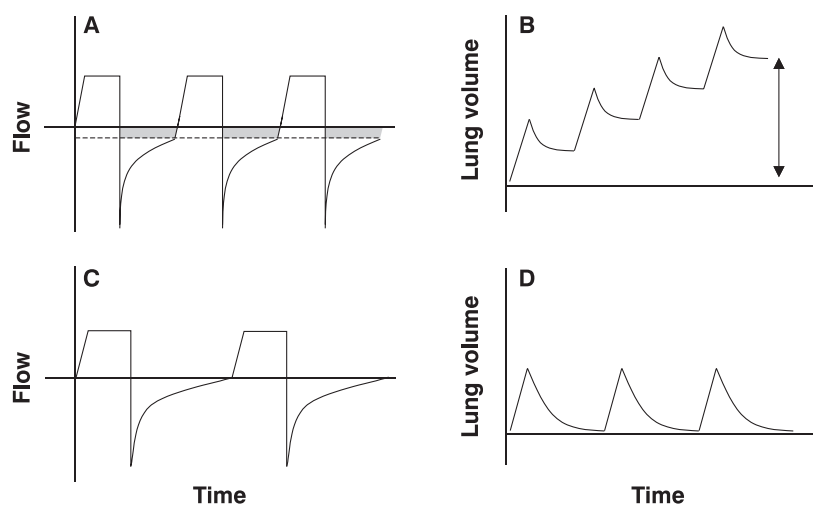

Figure 2 - Mechanics of dynamic pulmonary hyperinflation in the setting of severe airflow obstruction. A) A flow-time curve shows that inspiration begins before complete exhalation of the previous breath, leading to gas trapping (shaded area). B) Gas trapping over the course of several breaths leads to an increased end-expiratory lung volume. The trapped volume (Vtrap) over functional residual capacity is indicated by the doubleheaded arrow. C) Increase in expiratory time and decrease in respiratory rate allow for complete exhalation prior to the initiation of the next breath. D) Increase in expiratory time and decrease in respiratory rate result in no air trapping and absence of dynamic hyperinflation

The use of high-level pressure support in the management of spontaneously breathing intubated asthmatics or via face mask (BiPAP) has become common in recent years. The goal of this approach is the reduction in inspiratory work while allowing the patient to actively assist with exhalation. This is an intriguing strategy that warrants further study. ${ }^{18}$ Experience with children suffering from acute exacerbations of obstructive processes such as asthma, bronchiolitis and other conditions associated with reactive airway disease has demonstrated its ability to reduce the work of breathing, improve the delivery of inhaled bronchodilators and make many children subjectively more comfortable. In many cases, non-invasive positive pressure ventilation (NIPPV) appears to have avoided the need to intubate, however, it should be used only in facilities where intubation can be provided if needed.

\section{Mechanical ventilation in diseases of abnormal lung compliance}

Several disease processes highly prevalent in the PICU share abnormal lung compliance as a common feature, including lung disease due to surfactant deficiency of prematurity, large consolidated pneumonias, pulmonary edema, acute lung injury (ALI) and its more severe counterpart, the acute respiratory distress syndrome
(ARDS). While the abnormal lung compliance observed in premature infants with surfactant deficiency can be challenging to manage, the administration of exogenous surfactant can significantly improve the biomechanical properties of the lung, thus facilitating mechanical ventilation and minimizing secondary injury. In contrast, patients with diseases characterized by surfactant dysfunction and severe inflammation, such as those with pneumonias, postobstructive pulmonary edema, ALI and ARDS are less likely to benefit from surfactant replacement and require heightened attention in the choice of mechanical ventilation strategies due to the inhomogeneous nature of these diseases, i.e. vastly different mechanical properties of the dependent and non-dependent lung regions.

ARDS is the prototypical disease of abnormal lung compliance causing respiratory failure in both children and adults, ${ }^{19}$ excluding the premature infant. It is marked by a severe inflammatory response to local (pulmonary) or remote (systemic) insults that invariably leads to abnormalities in gas exchange and pulmonary mechanics. ${ }^{19}$ By definition, ARDS is characterized by the acute onset of severe hypoxemia $\left(\mathrm{PaO}_{2} / \mathrm{FiO}_{2}\right.$ ratio $\left.<200\right)$ and bilateral infiltrates on chest radiograph without evidence of increased left atrial pressure ${ }^{20}$ (pulmonary capillary wedge pressure < $18 \mathrm{mmHg}$ ). ARDS is associated with significant mortality ranging between 35 and $71 \%{ }^{5,21,22}$ and there is no curative treatment on the horizon. As a result of ventilation strategies developed over the last 15 years and the development of high-frequency oscillatory ventilation (HFOV), children tend to have a better prognosis for recovery from ARDS than adults.

In contrast to the homogenous, diffuse infiltrate seen on chest radiograph in ARDS, the actual distribution of lung injury is largely inhomogeneous, with inflammation, atelectasis and consolidation affecting primarily the gravitationally dependent lung while the non-dependent lung regions are largely spared ${ }^{23,24}$ (Figure 3 ). It is the inhomogeneous distribution of lung disease that makes mechanical ventilation in ARDS so challenging, as one may contribute unintentionally to further lung damage in what has been called ventilator-associated or ventilator-induced lung injury (VILI). ${ }^{1}$

Rather than attempt to discuss or recommend specific ventilator settings for what is a very dynamic disease process, we believe that lung protective ventilator management strategies for ARDS can best be developed by understanding the physical and biological processes involved in the genesis of VILI. 


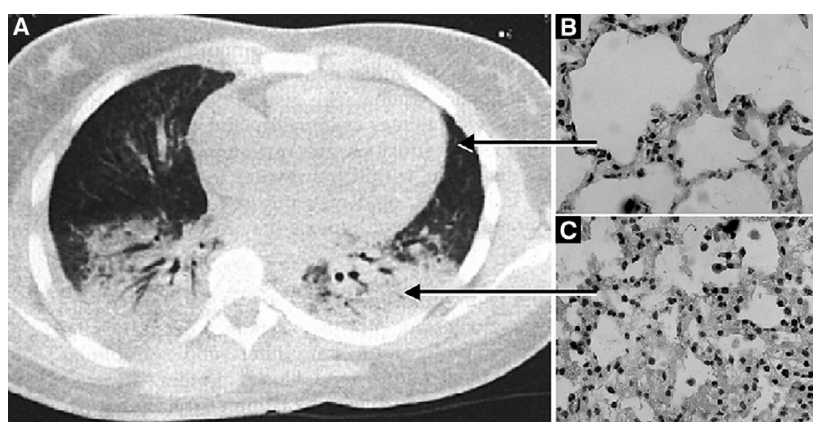

Figure 3 - A) Computed tomography of a patient with ARDS and photomicrographs of lung tissue showing $B$ ) the heterogeneous distribution of lung injury with relative sparing of the non-dependent region and C) inflammatory infiltration, edema, exudates and atelectasis of the dependent region

\section{Barotrauma vs. volutrauma}

Experimental data from animal studies have demonstrated that ventilation of healthy lungs with high inspiratory pressures leads to endothelial and epithelial cell damage and alterations in capillary permeability that result in non-hydrostatic pulmonary edema. ${ }^{25,26}$ The ensuing injury, originally called barotrauma, is directly proportional to the peak inspiratory pressure employed and to the duration of exposure to high pressures. ${ }^{26}$ However, further analysis clearly demonstrates that human lungs are capable of tolerating exposure to very high airway pressures as is the case of trumpet players that are repeatedly subjected to airway pressures in excess of $140 \mathrm{cmH}_{2} \mathrm{O}$ without developing injury. ${ }^{27}$ This phenomenon can be explained by the fact that although airway pressure might be very high, the musician has to contract his or her muscles to generate a high pleural pressure, so the actual transpulmonary pressure (alveolar minus pleural pressure) is not elevated. ${ }^{28}$ It has become clear that the lung distension caused by elevated transpulmonary pressures, not the high airway pressures themselves, is the major determinant in the development of VILI.

The relative individual role of tidal volume and peak inspiratory pressure in the genesis of VILI has been further clarified by the observation that mechanical ventilation with high or low tidal volumes, but with identical high peak airway pressures does not result in the same degree of lung abnormalities. ${ }^{2}$ Evidence of lung injury is found only in animals subjected to high peak inspiratory pressures and high tidal volume, but not in those in which lung distention was limited by thoracoabdominal strapping. ${ }^{2}$ Furthermore, animals ventilated with high tidal volumes and negative (and therefore low) airway pressures by means of an iron lung still developed increased capillary permeability and pulmonary edema, ${ }^{2}$ demonstrating that excessive tidal volume leading to regional overdistension, and not airway pressure, is the causal factor of this type of injury now termed volutrauma.
In ARDS, a large portion of the dependent lung is consolidated or has markedly decreased compliance and may not be available to participate in gas exchange. ${ }^{23,24}$ As a result, even tidal volumes of 10 to $12 \mathrm{~mL} / \mathrm{kg}$ can lead to significant overdistension and injury of the healthier and more compliant non-dependent lung regions through the maldistribution of the tidal volume to the more compliant regions.

\section{Atelectrauma}

In ARDS, alveoli in the dependent lung regions exhibit greatly reduced compliance compared to non-dependent alveoli. ${ }^{29}$ The more dependent alveoli often reach a critical closing volume at end-expiration resulting in alveolar collapse and airway closure that is followed by reopening of these alveoli and airways during inspiration. The cyclic repetition of alveolar collapse and reopening generates mechanical shearing forces capable of causing cumulative tissue damage, a phenomenon now termed atelectrauma. ${ }^{28}$

The application of PEEP can help stabilize diseased alveoli during expiration. The use of inadequately low PEEP that is insufficient to prevent the collapse of less compliant alveoli at end-expiration results in atelectrauma, while the use of adequate levels of PEEP that maintain an acceptable lung volume during exhalation is associated with more favorable physiological outcomes. ${ }^{3,30,31}$

\section{Biotrauma}

Experimental studies have shown that the detrimental effects of VILI go beyond the simple mechanical disruption of the pulmonary epithelial and endothelial surfaces. ${ }^{4}$ The use of a mechanical ventilation strategy that causes volutrauma (high tidal volume) and atelectrauma (zero PEEP) has been shown to promote the release of inflammatory mediators such as tumor necrosis factor alpha (TNF- $\alpha){ }^{32}$ This is in sharp contrast to the attenuated release of such mediators when a protective strategy with reduced tidal volume and high PEEP is employed. ${ }^{32}$

Injurious ventilation strategies may also lead to the release of cytokines and even bacteria from the lung into the systemic circulation, suggesting that the choice of mechanical ventilation strategy may impact not only the lung, but also adversely affect other organs. ${ }^{33}$ The remote effect of inflammatory mediators released by the lung has been the basis for theories linking non-protective ventilator strategies in patients with ARDS to the subsequent development of multi-organ system dysfunction, ${ }^{28}$ although this paradigm is still the object of significant debate. ${ }^{34}$

\section{Conventional lung protective ventilation}

Considering the strong experimental evidence discussed above, the application of a lung protective strategy in patients 
with ARDS using a conventional ventilator should be based on the use of reduced tidal volumes to avoid volutrauma and the use of adequate PEEP to prevent atelectrauma. Such an approach would theoretically promote ventilation in a "safe zone" (Figure 4).

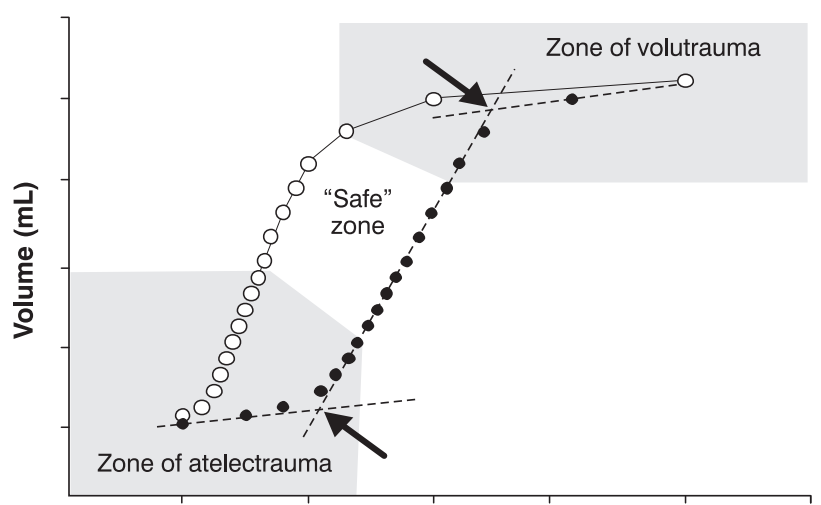

Pressure $\left(\mathrm{cmH}_{2} \mathrm{O}\right)$

Figure 4 - Static volume-pressure relationship of the respiratory system in an experimental model of ARDS, indicating the zones of ventilator-induced lung injury and the theoretical safe zone. The arrows indicate the lower and upper inflection points

The most robust evidence that using a lung protective ventilation strategy is advantageous in ARDS comes from studies in the adult population.5,35 However, when one considers the totality of available experimental and clinical data, there is no reason to believe that the same principles do not apply to children with ARDS.

A number of uncontrolled studies have reported lower-than-expected mortality rates in adult patients with severe ARDS treated with a strategy that limits peak inspiratory pressure by reducing tidal volume and allows for the development of permissive hypercapnia. ${ }^{36-39} \mathrm{~A}$ randomized multicenter trial has shown a significant reduction in mortality rates (from 39.8 to $31 \%$ ) in patients with ARDS managed with a reduced tidal volume strategy. ${ }^{35}$ Another randomized trial showed a dramatic decrease in mortality in the intervention group managed with limited inspiratory pressures, reduced tidal volume, lung recruitment maneuvers and the application of adequate levels of PEEP compared to a control group where no attempts were made to avoid VILI (38 vs. $71 \%$, respectively). ${ }^{5}$ Three other randomized trials failed to demonstrate a survival advantage associated with the use of a lung protective strategy based on reduced tidal volumes. ${ }^{40-42}$ In those trials, however, most patients in the control groups were subjected to ventilator settings unlikely to cause significant volutrauma. ${ }^{40-42}$
Until more definitive data become available for the pediatric ARDS population, it is reasonable to adopt a strategy that avoids volutrauma by employing reduced tidal volumes ( 5 to $8 \mathrm{~mL} / \mathrm{kg}$ ) to limit inspiratory plateau pressures, while utilizing adequate PEEP to reduce the likelihood of atelectrauma and minimize the exposure to hyperoxia through a reduction in intrapulmonary shunting.

\section{Permissive hypercapnia}

Permissive hypercapnia can be defined as the presence of an elevated $\mathrm{PaCO}_{2}$ in the setting of a mechanically ventilated patient receiving lung protective ventilation with limited inspiratory pressures and reduced tidal volumes. ${ }^{43}$ The resulting hypercapnia, which may reach blood levels over twice normal, is tolerated but not necessarily the goal of therapy, although experimental data suggest that hypercapnic acidosis might be associated with improved physiological outcomes. . $^{3,43-45}$

Permissive hypercapnia as part of a ventilation strategy tailored to minimize VILI was first used over 20 years ago in neonates with severe respiratory failure and pulmonary hypertension. ${ }^{46}$ Since then reports from various centers indicate that this approach can be successful in managing infants with severe respiratory failure and pulmonary hypertension due to congenital diaphragmatic hernia or meconium aspiration syndrome, while minimizing the need for extracorporeal membrane oxygenation (ECMO) and morbidity related to aggressive ventilatory therapy. $47-49$

Hickling at al. ${ }^{38}$ were the first to report a lower-than-expected mortality in adult patients with ARDS managed with a low tidal volume, pressure-limited ventilation strategy associated with permissive hypercapnia. A number of uncontrolled studies followed, reporting mortality rates between 0 and $30 \%$ in both adults and children subjected to a similar approach. ${ }^{39,50,51}$

Amato et al. ${ }^{5}$ demonstrated a significant reduction in mortality in patients receiving lung protective ventilation with reduced tidal volumes $(<6 \mathrm{~mL} / \mathrm{kg}$ ) compared to a so-called conventional approach $(12 \mathrm{~mL} / \mathrm{kg})$. The lung protective group was also subjected to lung volume optimization through recruitment maneuvers and the application of adequate PEEP to prevent derecruitment, i.e. "open lung" approach, and permissive hypercapnia. The study design does not allow one to determine what factor or factors were primarily responsible for the positive outcomes. Considering that the lung protective group ${ }^{5}$ was treated with a ventilation strategy that avoided volutrauma and atelectrauma, while optimizing lung recruitment and potentially reducing biotrauma, it is impossible to discern whether permissive 
hypercapnia was also an important factor contributing to the observed outcome or simply an epiphenomenon.

In the multicenter, randomized, controlled trial conducted by the ARDS Network ${ }^{35}$ comparing high $(12 \mathrm{~mL} / \mathrm{kg}$ ) and low $(6 \mathrm{~mL} / \mathrm{kg})$ tidal volumes, PEEP levels were similar between the two groups and most patients in the low tidal group did not develop hypercapnia as a result of an observed increase in the respiratory rate. As mentioned previously, the group treated with low tidal volumes had a significantly lower mortality compared to the high tidal volume group, ${ }^{35}$ a finding that was originally attributed solely to the effect of reduced lung stretch. Interestingly, there is now evidence that permissive hypercapnia could have played a role in attenuating high stretch lung injury in the ARDS Network trial. Using multivariate logistic regression, and controlling for comorbidities and severity of lung injury in the ARDS Network trial, $^{35}$ Kregenow et al. ${ }^{52}$ demonstrated that hypercapnic acidosis was associated with reduced 28-day mortality in the $12 \mathrm{~mL} / \mathrm{kg}$ tidal volume group in a dose-response fashion. These results were consistent with a protective effect of hypercapnic acidosis in and of itself against ventilatorassociated lung injury. This protection, however, was not observed when further ongoing injury was attenuated by the use of a low stretch strategy as in the group treated with reduced $(6 \mathrm{~mL} / \mathrm{kg})$ tidal volumes. ${ }^{52}$

\section{Advanced therapies}

Some patients with severe forms of ARDS will continue to deteriorate, despite concerted, well coordinated care and attempts to apply lung protective ventilation settings. Those with the most significant abnormalities of lung compliance and gas exchange simply may not tolerate ventilation at reduced tidal volumes and high PEEP, developing increased inspiratory pressures, profound acidosis and hemodynamic instability. For these patients, a trial of less conventional therapies such as high-frequency oscillatory ventilation (HFOV), prone positioning, inhaled nitric oxide, exogenous surfactant or ECMO might be of some additional value. However, a more extensive discussion of these treatment modalities is beyond the scope of this text.

\section{Conclusions}

It is clear that mechanical ventilation strategies influence the course of lung disease. A ventilation strategy that avoids dynamic hyperinflation and tolerates hypercapnia is the cornerstone of mechanical support of pediatric patients with diseases of abnormal airway resistance, such as near-fatal asthma. ${ }^{6}$

A ventilation strategy that avoids volutrauma, atelectrauma and biotrauma in the treatment of patients with diseases of abnormal lung compliance, such as ARDS, is firmly based on experimental literature and clinical experience. ${ }^{2-5,28,30,32}$ The application of a lung protective strategy incorporating reduced tidal volumes, effective lung recruitment, adequate PEEP to minimize alveolar collapse during expiration and permissive hypercapnia has been shown to be advantageous in adult patients with ARDS, ${ }^{5}$ although it has not been systematically studied in children.

The use of permissive hypercapnia as part of a lung protective strategy in children should be accepted and perhaps is desirable, provided it does not result in significant hemodynamic instability ${ }^{43}$ or hypoxemia.

\section{References}

1. Rotta AT, Kunrath $\mathrm{CL}$, Wiryawan B. [Management of the acute respiratory distress syndrome]. J Pediatr (Rio J). 2003;79 Suppl 2:S149-60.

2. Dreyfuss D, Soler P, Basset G, Saumon G. High inflation pressure pulmonary edema. Respective effects of high airway pressure, high tidal volume, and positive end-expiratory pressure. Am Rev Respir Dis. 1988;137:1159-64.

3. Rotta AT, Gunnarsson B, Fuhrman BP, Hernan LJ, Steinhorn DM. Comparison of lung protective ventilation strategies in a rabbit model of acute lung injury. Crit Care Med. 2001;29:2176-84.

4. Fu Z, Costello ML, Tsukimoto $K$, Prediletto R, Elliott AR, Mathieu-Costello $O$, et al. High lung volume increases stress failure in pulmonary capillaries. J Appl Physiol. $1992 ; 73: 123-33$.

5. Amato MB, Barbas CS, Medeiros DM, Magaldi RB, Schettino GP, Lorenzi-Filho $\mathrm{G}$, et al. Effect of a protective-ventilation strategy on mortality in the acute respiratory distress syndrome. N Engl J Med. 1998;338:347-54.

6. Rotta AT. Asthma. In: Fuhrman BP, Zimmerman J, editors. Pediatric critical care. Philadelphia: Mosby; 2006. p. 588-607.

7. Afessa B, Morales I, Cury JD. Clinical course and outcome of patients admitted to an ICU for status asthmaticus. Chest. 2001;120:1616-21.

8. Yung $M$, South M. Randomised controlled trial of aminophylline for severe acute asthma. Arch Dis Child. 1998;79:405-10.

9. Bohn D, Kissoon N. Acute asthma. Pediatr Crit Care Med. 2001;2:151-63.

10. Scoggin $\mathrm{CH}$, Sahn SA, Petty TL. Status asthmaticus. A nine-year experience. JAMA. 1977;238:1158-62.

11. Westerman DE, Benatar SR, Potgieter PD, Ferguson AD. Identification of the high-risk asthmatic patient. Experience with 39 patients undergoing ventilation for status asthmaticus. Am J Med. 1979;66:565-72.

12. Picado C, Montserrat JM, Roca J, Rodriguez-Roisin R, Estopa R, Xaubet $A$, et al. Mechanical ventilation in severe exacerbation of asthma. Study of 26 cases with six deaths. Eur J Respir Dis. $1983 ; 64: 102-7$.

13. Darioli R, Perret C. Mechanical controlled hypoventilation in status asthmaticus. Am Rev Respir Dis. 1984;129:385-7. 
14. Cox RG, Barker GA, Bohn DJ. Efficacy, results, and complications of mechanical ventilation in children with status asthmaticus. Pediatr Pulmonol. 1991;11:120-6.

15. Marini JJ. Should PEEP be used in airflow obstruction? Am Rev Respir Dis. 1989;140:1-3.

16. Stewart TE, Slutsky AS. Occult, occult auto-PEEP in status asthmaticus. Crit Care Med. 1996;24:379-80.

17. Tuxen DV. Detrimental effects of positive end-expiratory pressure during controlled mechanical ventilation of patients with severe airflow obstruction. Am Rev Respir Dis. 1989;140:5-9.

18. Wetzel RC. Pressure-support ventilation in children with severe asthma. Crit Care Med. 1996;24:1603-5.

19. Ware LB, Matthay MA. The acute respiratory distress syndrome. N Engl J Med. 2000;342:1334-49.

20. Bernard GR, Artigas A, Brigham KL, Carlet J, Falke K, Hudson L, et al. The American-European Consensus Conference on ARDS. Definitions, mechanisms, relevant outcomes, and clinical trial coordination. Am J Respir Crit Care Med. 1994;149:818-24.

21. Milberg JA, Davis DR, Steinberg KP, Hudson LD. Improved survival of patients with acute respiratory distress syndrome (ARDS): 1983-1993. JAMA. 1995;273:306-9.

22. Suchyta MR, Clemmer TP, Orme JF, Jr., Morris AH, Elliott CG. Increased survival of ARDS patients with severe hypoxemia (ECMO criteria). Chest. 1991;99:951-5.

23. Gattinoni L, Pesenti A, Bombino M, Baglioni S, Rivolta M, Rossi F, et al. Relationships between lung computed tomographic density, gas exchange, and PEEP in acute respiratory failure. Anesthesiology. 1988;69:824-32.

24. Gattinoni L, Presenti A, Torresin A, Baglioni S, Rivolta M, Rossi F, et al. Adult respiratory distress syndrome profiles by computed tomography. J Thorac Imaging. 1986;1:25-30.

25. Dreyfuss D, Basset G, Soler P, Saumon G. Intermittent positivepressure hyperventilation with high inflation pressures produces pulmonary microvascular injury in rats. Am Rev Respir Dis. $1985 ; 132: 880-4$.

26. Webb HH, Tierney DF. Experimental pulmonary edema due to intermittent positive pressure ventilation with high inflation pressures. Protection by positive end-expiratory pressure. Am Rev Respir Dis. 1974;110:556-65.

27. Bouhuys A. Physiology and musical instruments. Nature. 1969;221:1199-204.

28. Slutsky AS. Ventilator-induced lung injury: from barotrauma to biotrauma. Respir Care. 2005;50:646-59.

29. Gattinoni L, D'Andrea L, Pelosi P, Vitale G, Pesenti A, Fumagalli R. Regional effects and mechanism of positive end-expiratory pressure in early adult respiratory distress syndrome. JAMA. 1993;269:2122-7.

30. Muscedere JG, Mullen JB, Gan K, Slutsky AS. Tidal ventilation at low airway pressures can augment lung injury. Am J Respir Crit Care Med. 1994;149:1327-34.

31. Viana ME, Sargentelli GA, Arruda AL, Wiryawan B, Rotta AT. [The impact of mechanical ventilation strategies that minimize atelectrauma in an experimental model of acute lung injury]. J Pediatr (Rio J). 2004;80:189-96.

32. Tremblay LN, Miatto D, Hamid Q, Govindarajan A, Slutsky AS. Injurious ventilation induces widespread pulmonary epithelial expression of tumor necrosis factor-alpha and interleukin-6 messenger RNA. Crit Care Med. 2002;30:1693-700.
33. Chiumello D, Pristine G, Slutsky AS. Mechanical ventilation affects local and systemic cytokines in an animal model of acute respiratory distress syndrome. Am J Respir Crit Care Med. 1999;160:109-16.

34. Dreyfuss D, Ricard JD, Saumon G. On the physiologic and clinical relevance of lung-borne cytokines during ventilator-induced lung injury. Am J Respir Crit Care Med. 2003;167:1467-71.

35. ARDS-Network. Ventilation with lower tidal volumes as compared with traditional tidal volumes for acute lung injury and the acute respiratory distress syndrome. The acute respiratory distress syndrome network. N Engl J Med. 2000;342:1301-8.

36. Froese AB, Bryan AC. High frequency ventilation. Am Rev Respir Dis. 1987; $135: 1363-74$.

37. Hickling KG. Permissive hypercapnia. Respir Care Clin N Am. 2002;8:155-69, v.

38. Hickling KG, Henderson SJ, Jackson R. Low mortality associated with low volume pressure limited ventilation with permissive hypercapnia in severe adult respiratory distress syndrome. Intensive Care Med. 1990;16:372-7.

39. Hickling KG, Walsh J, Henderson S, Jackson R. Low mortality rate in adult respiratory distress syndrome using low-volume, pressure-limited ventilation with permissive hypercapnia: a prospective study. Crit Care Med. 1994;22:1568-78.

40. Brochard L, Roudot-Thoraval F, Roupie E, Delclaux C, Chastre J, Fernandez-Mondejar $\mathrm{E}$, et al. Tidal volume reduction for prevention of ventilator-induced lung injury in acute respiratory distress syndrome. The Multicenter Trial Group on Tidal Volume reduction in ARDS. Am J Respir Crit Care Med. 1998; 158:1831-8.

41. Brower RG, Shanholtz CB, Fessler HE, Shade DM, White P Jr., Wiener $\mathrm{CM}$, et al. Prospective, randomized, controlled clinical trial comparing traditional versus reduced tidal volume ventilation in acute respiratory distress syndrome patients. Crit Care Med. 1999;27:1492-8.

42. Stewart TE, Meade MO, Cook DJ, Granton JT, Hodder RV, Lapinsky SE, et al. Evaluation of a ventilation strategy to prevent barotrauma in patients at high risk for acute respiratory distress syndrome. Pressure- and Volume-Limited Ventilation Strategy Group. N Engl J Med. 1998;338:355-61.

43. Rotta AT, Steinhorn DM. Is permissive hypercapnia a beneficial strategy for pediatric acute lung injury? Respir Care Clin N Am. 2006;12:371-87.

44. Laffey JG, Honan D, Hopkins N, Hyvelin JM, Boylan JF, McLoughlin P. Hypercapnic acidosis attenuates endotoxininduced acute lung injury. Am J Respir Crit Care Med. 2004; 169:46-56.

45. Laffey JG, O'Croinin D, McLoughlin P, Kavanagh BP. Permissive hypercapnia--role in protective lung ventilatory strategies. Intensive Care Med. 2004;30:347-56.

46. Wung JT, James LS, Kilchevsky E, James E. Management of infants with severe respiratory failure and persistence of the fetal circulation, without hyperventilation. Pediatrics. $1985 ; 76: 488-94$.

47. Dworetz AR, Moya FR, Sabo B, Gladstone I, Gross I. Survival of infants with persistent pulmonary hypertension without extracorporeal membrane oxygenation. Pediatrics. $1989 ; 84: 1-6$.

48. Ivascu FA, HirschI RB. New approaches to managing congenital diaphragmatic hernia. Semin Perinatol. 2004;28:185-98. 
49. Boloker J, Bateman DA, Wung JT, Stolar CJ. Congenital diaphragmatic hernia in 120 infants treated consecutively with permissive hypercapnia/spontaneous respiration/elective repair. J Pediatr Surg. 2002;37:357-66.

50. Lewandowski K, Rossaint R, Pappert D, Gerlach H, Slama KJ, Weidemann $\mathrm{H}$, et al. High survival rate in 122 ARDS patients managed according to a clinical algorithm including extracorporeal membrane oxygenation. Intensive Care Med. $1997 ; 23: 819-35$.

51. Nakagawa S, Bohn D. Pressure controlled ventilation with limited peak inspiratory pressure bellow 35 to $40 \mathrm{~cm} \mathrm{H}_{2} \mathrm{O}$ may improve survival of pediatric acute respiratory failure. Am J Respir Crit Care Med. 1995;151:A77.
52. Kregenow DA, Rubenfeld GD, Hudson LD, Swenson ER. Hypercapnic acidosis and mortality in acute lung injury. Crit Care Med. 2006;34:1-7.

Correspondence:

Alexandre T. Rotta

Department of Anesthesiology

3533 S. Alameda Street

78411 Corpus Christi, TX - USA

Tel.: +1 (361) 694.5445

Fax: +1 (361) 694.5449

E-mail: alexrotta@stx.rr.com 